\title{
SPACE FILLING CURVES OVER FINITE FIELDS
}

\author{
NiCHOLAS M. KATZ
}

\section{Introduction}

In this note, we construct curves over finite fields which have, in a certain sense, a "lot" of points, and give some applications to the zeta functions of curves and abelian varieties over finite fields. In fact, we found the basic construction, given in Lemma 1, of curves in $\mathbb{A}^{n}$ which go through every rational point, as part of an unsuccessful attempt to find curves of growing genus over a fixed finite field with lots of points in the sense of the Drinfield-Vladut bound [2]. The idea of applying that construction along the lines of this note grew out of an August 1996 conversation with Ofer Gabber about whether every abelian variety over a finite field was a quotient of a Jacobian, during which he constructed, on the fly, a proof of that fact. A variant of his proof appears here in Theorem 11. It is a pleasure to acknowledge my debt to him.

\section{The basic constructions}

Lemma 1. Let $k$ be a finite field, $p$ its characteristic, $\bar{k}$ an algebraic closure of $k, E / k$ a finite extension inside $\bar{k}$, and $n \geq 1$ an integer. There exists a smooth, geometrically connected curve $C / k$ and a closed immersion of $k$-schemes

$$
C \subset \mathbb{A}^{n} \otimes_{\mathbb{Z}} k
$$

which induces a bijection of E-valued points

$$
C(E)=\mathbb{A}^{n}(E) .
$$

Construction-proof. If $n=1$, take $C=\mathbb{A}^{n} \otimes_{\mathbb{Z}} k$. If $n=r+1$ with $r \geq 1$, choose a sequence of $r$ nonzero polynomials in one variable over $k, f_{1}(X), \ldots, f_{r}(X)$, with the following three properties:

1) For each $i, f_{i}(x)=0$ for every $x \in E$.

2) For each $i$, the degree $d_{i}$ of $f_{i}$ is prime to $p$.

3) The degrees are strictly increasing: $d_{1}<d_{2}<\cdots<d_{r}$.

[Here is a simple way to make such a choice. Write $q:=\# E$, and pick a strictly increasing sequence of $r$ positive integers each of which is prime to $p$, say $e_{1}<e_{2}<\cdots<e_{r}$. Then take each $f_{i}(X):=\left(X^{q}-X\right) X^{e_{i}}$.]

Received March 4, 1999. 
In $\mathbb{A}^{r+1} \otimes k$ with coordinates $X, Y_{1}, \ldots, Y_{r}$, consider the closed subscheme $C / k$ defined by the $r$ equations

$$
\left(Y_{i}\right)^{q}-Y_{i}=f_{i}(X), \quad i=1, \ldots, r .
$$

It is obvious from these equations that every $E$-valued point of $\mathbb{A}^{n}$ lies in $C$. We must see that $C / k$ is a smooth curve which is geometrically connected.

First of all, $C / k$ is a smooth curve, for it is the fibre product over $\mathbb{A}^{1} \otimes k$ of $r$ finite etale galois coverings $\mathcal{E}_{i} \rightarrow \mathbb{A}^{1} \otimes k$, with $\mathcal{E}_{i}$ the affine plane curve $(Y)^{q}-Y=f_{i}(X)$ in $\mathbb{A}^{2} \otimes k$.

It remains to see that $C \otimes_{k} \bar{k}$ is connected. This results from Artin-Schreier theory. On $\mathbb{A}^{1} \otimes \bar{k}$, or indeed on any smooth, affine, connected scheme $S / \bar{k}$, the Artin-Schreier sequence relative to $q$,

$$
0 \rightarrow E \rightarrow \mathcal{O}_{S} \stackrel{f \mapsto \mathcal{P}(f):=f^{q}-f}{\longrightarrow} \mathcal{O}_{S} \rightarrow 0
$$

gives, via the long exact cohomology sequence, an isomorphism of $E$-vector spaces

$$
H^{0}\left(S, \mathcal{O}_{S}\right) / \mathcal{P}\left(H^{0}\left(S, \mathcal{O}_{S}\right)\right) \cong H_{\text {et }}^{1}(S, E)=\operatorname{Hom}\left(\pi_{1}(S), E\right) .
$$

Given $f$ in $H^{0}\left(S, \mathcal{O}_{S}\right)$, the covering of $S$ defined by $Y^{q}-Y=f\left(\right.$ in $\left.\mathbb{A}^{1} \times S\right)$ is finite etale galois with group $E$ ( $\alpha$ in $E$ translates $Y$ ), so "is" an element Class $(f)$ in $\operatorname{Hom}\left(\pi_{1}(S), E\right)$.

Now return to the case when $S$ is $\mathbb{A}^{1} \otimes \bar{k}$ and take any nontrivial $\mathbb{C}$-valued character $\psi$ of $E$. If $f$ in $\bar{k}[X]$ has degree $d$ prime to $p$, then the composite homomorphism is known $[1,3.5 .4]$ to have Swan conductor $d$ at $\infty$.

Our $C \otimes_{k} \bar{k}$ is a finite etale galois covering of $\mathbb{A}^{1} \otimes \bar{k}$ with group $E \times E \times \cdots \times E=$ $E^{r}$, corresponding to the $r$-tuple $\left(f_{1}, f_{2}, \ldots, f_{r}\right)$ via

$$
(\bar{k}[X] / \mathcal{P}(\bar{k}[X]))^{r} \cong H_{\mathrm{et}}^{1}\left(\mathbb{A}^{1} \otimes \bar{k}, E^{r}\right)=\operatorname{Hom}\left(\pi_{1}(S), E^{r}\right) .
$$

The total space $C \otimes_{k} \bar{k}$ of this covering is connected if and only if the corresponding homomorphism

$$
\operatorname{Class}\left(f_{1}, f_{2}, \ldots, f_{r}\right): \pi_{1}\left(\mathbb{A}^{1} \otimes \bar{k}\right) \rightarrow E^{r}
$$

is surjective, or equivalently (Pontrajagin duality!) if and only if for every nontrivial $\mathbb{C}$-valued additive character $\left(\psi_{1}, \psi_{2}, \ldots, \psi_{r}\right)$ of $E^{r}$, the composite homomorphism

$$
\left(\psi_{1}, \psi_{2}, \ldots, \psi_{r}\right) \circ \operatorname{Class}\left(f_{1}, f_{2}, \ldots, f_{r}\right): \pi_{1}\left(\mathbb{A}^{1} \otimes \bar{k}\right) \rightarrow \mathbb{C}^{\times},
$$

is nontrivial. But this composite is just the product

$$
\left(\psi_{1}, \psi_{2}, \ldots, \psi_{r}\right) \circ \operatorname{Class}\left(f_{1}, f_{2}, \ldots, f_{r}\right)=\prod_{i} \mathcal{L}_{\psi_{i}}\left(f_{i}\right) .
$$

In this product, $\mathcal{L}_{\psi_{i}}\left(f_{i}\right)$ is trivial if $\psi_{i}$ itself is trivial, and $\mathcal{L}_{\psi_{i}}\left(f_{i}\right)$ has $\operatorname{Swan}_{\infty}=$ $d_{i}$ if $\psi_{i}$ is nontrivial. Because the $d_{i}$ are all distinct, and at least one $\psi_{i}$ is nontrivial, we have

$$
\operatorname{Swan}_{\infty}\left(\prod_{i} \mathcal{L}_{\psi_{i}}\left(f_{i}\right)\right)=\operatorname{Sup}_{i \text { with } \psi_{i} \text { nontriv }}\left(d_{i}\right)>0 .
$$


Hence $\prod_{i} \mathcal{L}_{\psi_{i}}\left(f_{i}\right)$ must be nontrivial.

Lemma 2. Let $k$ be a finite field, $X / k$ projective (resp. quasi-projective), smooth, and geometrically connected of dimension $n \geq 1$. Let $E / k$ be a finite extension. There exists an affine (resp. quasi-affine) open set $U \subset X$ which contains all the E-valued points of $X$, i.e., $U(E)=X(E)$.

Proof. To fix ideas, say $X \subset \mathbb{P}^{N} \otimes k$. We need only construct an affine open set $U$ in $\mathbb{P}^{N} \otimes k$ which contains all the $E$-valued points of $\mathbb{P}^{N} \otimes k$, for then $X \cap U$ is the desired affine (resp. quasi-affine) open set of $X$. To do this, denote by $K / E$ the field extension of degree $N+1$, and pick a basis $\alpha_{0}, \alpha_{1}, \ldots, \alpha_{N}$ of $K / E$. Denote by $H$ the form of degree $N+1$ in $X_{0}, \ldots, X_{N}$ with coefficients in $E$ defined by

$$
H\left(X^{\prime} s\right):=\operatorname{Norm}_{K / E}\left(\alpha_{0} X_{0}+\cdots+\alpha_{N} X_{N}\right) .
$$

Then $H$ is nonzero at every $E$-valued point of $\mathbb{P}^{N}$. For each $\sigma$ in $\operatorname{Gal}(E / k)$, the form $H^{\sigma}$ has the same property (indeed, if we extend $\sigma$ to an element $\tilde{\sigma}$ in $\operatorname{Gal}(K / k)$ which induces $\sigma$, then $\tilde{\sigma}\left(\alpha_{0}, \alpha_{1}, \ldots, \alpha_{N}\right)$ is another basis of $K / E$, and $H^{\sigma}$ is its norm form to $\left.E\right)$. So $\operatorname{Norm}_{E / k}(H)$ is a form with coefficients in $k$ which is nonzero at every $E$-valued point of $\mathbb{P}^{N}$. We may take for $U$ the affine open set $\left(\mathbb{P}^{N} \otimes k\right)\left[1 / \operatorname{Norm}_{E / k}(H)\right]$.

Lemma 3. Let $k$ be a finite field, $U / k$ a quasi-affine, smooth, and geometrically connected of dimenstion $n \geq 1$. Let $E / k$ be a finite extension. There exists an open set $V \subset U$ which contains all the E-valued points of $U$ and which admits an etale map to $\mathbb{A}^{n} \otimes k$.

Proof. Say $U$ is open in the affine scheme $\bar{U}$. First view $U(E)$ as a finite closed subscheme $Z$ of $U$, by grouping its points into orbits under $\operatorname{Gal}(E / k)$. More precisely, $Z$ is the disjoint union of the finitely many closed points of $U$ the degree over $k$ of whose residue fields divides $\operatorname{deg}(E / k)$, with its reduced structure. Thus, $Z$ is a closed subscheme of $U$ which is finite etale over $k$. This same $Z$ is closed in $\bar{U}$, since we may describe it as the disjoint union of the finitely many closed points of $\bar{U}$ whose residue field degrees over $k$ divide $\operatorname{deg}(U / k)$ and which lie in $U$. Denote by $A$ the coordinate ring of $\bar{U}, I \subset A$ the ideal defining $Z$. At each point $P$ in $Z$, pass to the local ring $\mathcal{O}_{\bar{U}, P}$ of $P$ in $\bar{U}$, and pick $n$ elements $f_{1, P}, f_{2, P}, \ldots, f_{n, P}$ which form a $k(P)$-basis of $\mathbf{m} / \mathbf{m}^{2}, \mathbf{m}$ the maximal ideal. The ring $A / I^{2}$ is just the product ring $\prod_{P \in Z} \mathcal{O}_{\bar{U}, P} / \mathbf{m}^{2}$. So, we can find functions $f_{1}, \ldots, f_{n}$ in $A$ such that, for each $i$ and each $P, f_{i}$ induces $f_{i, P}$ in $\mathcal{O}_{\bar{U}, P} / \mathbf{m}^{2}$. Restrict each function $f_{i}$ to $U$, and view $\left(f_{1}, \ldots, f_{n}\right)$ as a map $\pi$ of $U$ to $\mathbb{A}^{n}$. This map $\pi$ is etale at each point $P$ in $Z$ by construction. Thus, the set $V$ of points of $U$ at which $\pi$ is etale is open, and contains $Z$.

Lemma 4. Let $k$ be a finite field, $V / k$ smooth and geometrically connected of dimension $n \geq 1$, and

$$
\pi: V \rightarrow \mathbb{A}^{n} \otimes k
$$


an etale map of $k$-schemes. For each integer $r \geq 1$, denote by $k_{r}$ the extension field of $k$ inside $\bar{k}$ of degree $r$ over $k$. For each $r \geq 1$, apply Lemma 1 with $E:=k_{r}$ to produce a closed immersion

$$
i_{r}: C_{r} / k \hookrightarrow \mathbb{A}^{n} \otimes k,
$$

with $C_{r} / k$ a smooth, geometrically connected curve such that

$$
C_{r}\left(k_{r}\right)=\mathbb{A}^{n}\left(k_{r}\right) .
$$

Form the fibre product

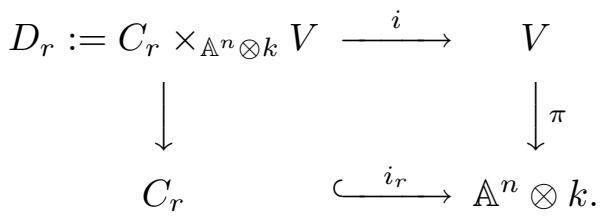

1) For every $r, D_{r} / k$ is a smooth curve, space-filling in $V$ for $k_{r}$, i.e., via the closed immersion

$$
i: D_{r}:=C_{r} \times_{\mathbb{A}^{n} \otimes k} V \longrightarrow V,
$$

we have

$$
D_{r}\left(k_{r}\right)=V\left(k_{r}\right) \text {. }
$$

2) For all sufficiently large $r, D_{r} / k$ is geometrically connected.

Proof. 1) is obvious from the cartesian diagram defining $D_{r}$, in which $\pi$ is etale, $C_{r} / k$ is a smooth curve, and $i_{r}$ is surjective on $k_{r}$-valued points.

To prove 2), we argue as follows. The etale map $\pi$ need not be finite etale, but there is a dense open set $j: W \hookrightarrow \mathbb{A}^{n} \otimes k$ over which $\pi$ is finite etale (just because $\pi$ is finite etale over the generic point of $\mathbb{A}^{n} \otimes k$ ). Take the entire diagram

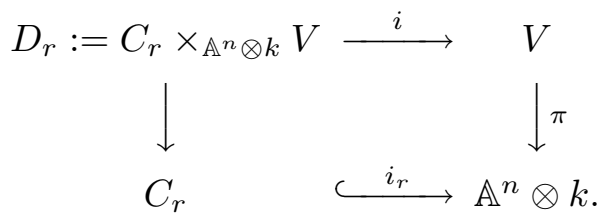

in the category of $\mathbb{A}^{n} \otimes k$-schemes, and pull it back to the open set $W$, i.e., base change it by $j: W \hookrightarrow \mathbb{A}^{n} \otimes k$. We get a diagram

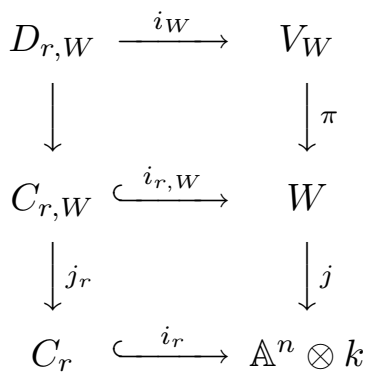


In this diagram, both $W$ and $V_{W}$ are smooth over $k$ and geometrically connected, $\pi$ is finite etale, and $i_{r, W}: C_{r, W} \hookrightarrow W$ is spacefilling for $k_{r}$. Now $C_{r, W}$ is open in $C_{r}$, so it is either dense and open in $C_{r}$ and itself geometrically connected, or it is empty. For large $r, C_{r, W}$ is not empty, because $W\left(k_{r}\right)$ is nonempty for large $r$ (by Lang-Weil, because $W / k$ is geometrically irreducible), and $i_{r, W}: C_{r, W} \hookrightarrow W$ is spacefilling for $k_{r}$. Let us temporarily admit the truth of

Lemma 5. Let $k$ be a finite field, $\mathcal{E} / k$ and $W / k$ two smooth, geometrically connected $k$-schemes of the same dimension $n \geq 1$, and

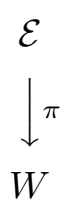

a finite etale $k$-morphism. Suppose given an integer $r_{0} \geq 1$, and for all integers $r \geq r_{0}$, a smooth, geometrically connected curve $\mathcal{C}_{r} / k$ and a closed $k$-immersion $i_{r}: \mathcal{C}_{r} \rightarrow W$ which is spacefilling for $k_{r}$, i.e., $\mathcal{C}_{r}\left(k_{r}\right)=W\left(k_{r}\right)$. Form the fibre product

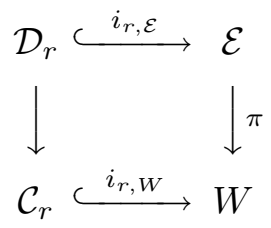

Then for $r$ sufficiently large, the curve $\mathcal{D}_{r} / k$ is geometrically connected.

Applying this lemma to our situation $\left(\mathcal{E}\right.$ is $V_{W}, \mathcal{C}_{r}$ is $\left.C_{r, W}\right)$, we find that for large $r, D_{r, W}$ is geometrically connected. We wish to infer that $D_{r} / k$ itself is geometrically connected. If it is not, then $D_{r} \otimes_{k} \bar{k}$ is a union of two or more connected components, each of which is etale over $C_{r} \otimes_{k} \bar{k}$. But as etale maps are open, the image of each connected component meets the dense open set $C_{r, W} \otimes_{k} \bar{k}$, and hence $D_{r, W} \otimes_{k} \bar{k}$ is not connected, contradiction. QED for Lemma 4 modulo Lemma 5.

Proof of Lemma 5. Fix a geometric point $\omega$ in $W \otimes_{k} \bar{k}$, and view the finite etale covering $\pi: \mathcal{E} \rightarrow W$ as an action of the group $\pi_{1}(W, \omega)$ on the finite set $S:=\pi^{-1}(\omega)$, i.e., a homomorphism

$$
\rho: \pi_{1}(W, \omega) \rightarrow \operatorname{Aut}(S) .
$$

The geometric connectedness of $\mathcal{E}$ means precisely that via this action, the subgroup

$$
\pi_{1}^{\text {geom }}(W, \omega):=\pi_{1}\left(W \otimes_{k} \bar{k}, \omega\right) \subset \pi_{1}(W, \omega)
$$

acts transitively on $S$. Recall the short exact sequence 


$$
1 \rightarrow \pi_{1}^{\text {geom }}(W, \omega) \rightarrow \pi_{1}(W, \omega) \stackrel{\text { degree }}{\longrightarrow} \operatorname{Gal}(\bar{k} / k) \rightarrow 1
$$

Denote by

$$
\Gamma_{\text {geom }} \subset \Gamma \subset \operatorname{Aut}(S)
$$

the images in $\operatorname{Aut}(S)$ of $\pi_{1}^{\text {geom }}(W, \omega)$ and of $\pi_{1}(W, \omega)$ respectively under $\rho$. The quotient $\Gamma / \Gamma_{\text {geom }}$ is cyclic, say of order $N$, generated by $\rho(F)$ for any fixed element $F$ in $\pi_{1}(W, \omega)$ of degree 1 . For each $i$ in $\mathbb{Z} / N \mathbb{Z}$, denote by $\Gamma(i) \subset \Gamma$ the set of elements whose degree $\bmod N$ is $i$, i.e., $\Gamma(i)$ is the coset $\rho\left(F^{i}\right) \Gamma_{\text {geom }}$.

By Chebotarev (cf., [5, 9.7.13]) for every $r \gg 0$, we have:

$(* * r, \mathcal{E} / W)$ The images under $\rho$ of all degree $r$ Frobenius elements in $\pi_{1}(W, \omega)$, i.e., all elements in all Frobenius conjugacy classes

$$
\text { Frob }_{k_{r}, w} \text { in } \pi_{1}(W, \omega)
$$

attached to $k_{r}$-valued points $w$ of $W$, fill the coset $\Gamma(r)$.

We will show that for any $r \geq r_{0}$ large enough that $(* * r, \mathcal{E} / W)$ holds, $\mathcal{D}_{r}$ is geometrically connected. To see this, pick a geometric point $c_{r}$ in $\mathcal{C}_{r}$, take for $\omega$ its image in $W$, and consider the composite homomorphism

$$
\pi_{1}\left(\mathcal{C}_{r}, c_{r}\right) \stackrel{\pi_{1}\left(i_{r, W}\right)}{\longrightarrow} \pi_{1}(W, \omega) \stackrel{\rho}{\longrightarrow} \Gamma \subset \operatorname{Aut}(S),
$$

which we label

$$
\rho_{r}: \pi_{1}\left(\mathcal{C}_{r}, c_{r}\right) \rightarrow \Gamma \subset \operatorname{Aut}(S) .
$$

Now $\mathcal{D}_{r} / k$ is geometrically connected if and only if the subgroup

$$
\rho_{r}\left(\pi_{1}^{\text {geom }}\left(\mathcal{C}_{r}, c_{r}\right)\right) \subset \operatorname{Aut}(S)
$$

acts transitively on $S$. A sufficient condition for this transitivity is that

$$
\rho_{r}\left(\pi_{1}^{\text {geom }}\left(\mathcal{C}_{r}, c_{r}\right)\right)=\Gamma_{\text {geom }},
$$

(because the geometric connectedness of $\mathcal{E}$ means that $\Gamma_{\text {geom }}$ acts transitively). A sufficient condition for

$$
\rho_{r}\left(\pi_{1}^{\text {geom }}\left(\mathcal{C}_{r}, c_{r}\right)\right)=\Gamma_{\text {geom }},
$$

is that the condition $\left(* * r, \mathcal{D}_{r}, \mathcal{C}_{r}\right)$ hold:

$\left(* * r, \mathcal{D}_{R}, \mathcal{C}_{r}\right)$ The images under $\rho_{r}$ of all the Frobenius elements of degree $r$ in $\pi_{1}\left(\mathcal{C}_{r}, c_{r}\right)$ fill $\Gamma(r)$.

Indeed, every element in $\Gamma_{\text {geom }}:=\Gamma(0)$ is of the form $A^{-1} B$ with $A$ and $B$ in $\Gamma(r)=\rho\left(F^{r}\right) \Gamma_{\text {geom }}$, and hence every element of $\Gamma_{\text {geom }}$ will be the image under $\rho_{r}$ of a ratio $\left(\operatorname{Frob}_{k_{r}, x}\right)^{-1}\left(\operatorname{Frob}_{k_{r}, y}\right)$ for two points $x$ and $y$ in $\mathcal{C}_{r}\left(k_{r}\right)$. Such a ratio lies in $\pi_{1}^{\text {geom }}\left(\mathcal{C}_{r}, c_{r}\right)$.

But $\mathcal{C}_{r}\left(k_{r}\right)=W\left(k_{r}\right)$ by assumption, so every degree $r$ Frobenius element in $\pi_{1}(W, \omega)$ is the image under $\pi_{1}\left(i_{r, W}\right)$ of a degree $r$ Frobenius element in 
$\pi_{1}\left(\mathcal{C}_{r}, c_{r}\right)$. Therefore $\left(* * r, \mathcal{D}_{r} / \mathcal{C}_{r}\right)$ is equivalent to $(* * r, \mathcal{E} / W)$. In particular, for large $r,\left(* * r, \mathcal{D}_{r} / \mathcal{C}_{r}\right)$ and hence $(* r)$ hold.

With an eye to later applications, we extract from the proof of Lemma 5 the following variant.

Lemma 6. Let $k$ be a finite field, $W / k$ a smooth, geometrically connected $k$ scheme, and $w$ a geometric point of $W$. Suppose given an integer $r_{0} \geq 1$, and, for each integer $r \geq r_{0}$, a smooth geometrically connected $k$-scheme $\mathcal{C}_{r} / k$ and a k-morphism

$$
f_{r}: \mathcal{C}_{r} \rightarrow W
$$

which is surjective on $k_{r}$-valued points. For each $r \geq r_{0}$, pick a geometric point $c_{r}$ in $\mathcal{C}_{r}$, and a "chemin" from $f_{r}\left(c_{r}\right)$ to $w$.

Suppose that $G$ is either

1) a finite group, or,

2) $\operatorname{GL}\left(n, \mathcal{O}_{\lambda}\right)$ for some positive integer $n$ and for $\mathcal{O}_{\lambda}$ the ring of integers in a finite extension of $\mathbb{Q}_{l}$, for some prime number $l$.

3) $\operatorname{GL}\left(n, \overline{\mathbb{Q}}_{l}\right)$ for some $n$ and some prime $l$.

Suppose given a continuous group homomorphism

$$
\rho: \pi_{1}(W, w) \rightarrow G .
$$

We denote

$$
\rho_{r}: \pi_{1}\left(\mathcal{C}_{r}, c_{r}\right) \rightarrow G
$$

the composite homomorphism

$$
\pi_{1}\left(\mathcal{C}_{r}, c_{r}\right) \stackrel{f_{*}}{\longrightarrow} \pi_{1}\left(W, f\left(c_{r}\right)\right) \stackrel{\text { chemin }}{\longrightarrow} \pi_{1}(W, w) \stackrel{\rho}{\longrightarrow} G .
$$

Then we have:

a) For $r$ sufficiently large, we have an equality of images of geometric fundamental groups

$$
\rho_{r}\left(\pi_{1}^{\text {geom }}\left(\mathcal{C}_{r}, c_{r}\right)\right)=\rho\left(\pi_{1}^{\text {geom }}(W, w)\right)
$$

(equality inside $G$ ).

b) Suppose in addition that, for each $r \geq r_{0}, f_{r}$ is also surjective on $k_{s}$-valued points for all divisors $s$ of $r$. Then for $r$ sufficiently large and sufficiently divisible, we have an equality of images of fundamental groups

$$
\rho_{r}\left(\pi_{1}\left(\mathcal{C}_{r}, c_{r}\right)\right)=\rho\left(\pi_{1}(W, w)\right)
$$

(equality inside $G$ ).

Proof. In case 1$), G$ finite, we put $\Gamma:=\rho\left(\pi_{1}(W, w)\right), \Gamma_{\text {geom }}:=\rho\left(\pi_{1}^{\text {geom }}(W, w)\right)$, denote by $N$ the order of the cyclic group $\Gamma / \Gamma_{\text {geom }}$, and denote by $\Gamma(i)$ the set of elements in $\Gamma$ of degree $i \bmod N$. By Chebotarev, for $r \gg 0$, the Frobenii of $k_{r}$-valued points of $W$ fill the coset $\Gamma(r)$, hence by the surjectivity of the map $f_{r}$ on $k_{r}$-valued points, so do the Frobenii of $k_{r}$-valued points of $\mathcal{C}_{r}$ for $r \gg 0$. For these $r$, the $A^{-1} B$ argument shows that ratios $A^{-1} B$ of such Frobenii fill $\Gamma_{\text {geom }}$, whence a). 
For b), we argue as follows. For each integer $i$ in $[0, N-1]$ pick an integer $d_{i} \equiv i \bmod N$ and sufficiently large that the Frobenii of $k_{d_{i}}$-valued points of $W$ fill the coset $\Gamma(i)$. Then for any $r \geq r_{0}$ which is divisible by $\prod_{i} d_{i}$, the Frobenii of the points on $\mathcal{C}_{r}$ with values in $k_{d_{i}}$ for $i=0,1, \ldots, N-1$ fill $\Gamma$.

For case 2$)$, put $K:=$ the image $\rho\left(\pi_{1}^{\text {geom }}(W, w)\right)$ in $\operatorname{GL}\left(n, \mathcal{O}_{\lambda}\right)$. By Pink's Lemma [4, 8.18.3], there exists an integer $d \geq 1$ such that a closed subgroup $H$ of $K$ is equal to $K$ if and only if $H$ and $K$ have the same image in $\operatorname{GL}\left(n, \mathcal{O}_{\lambda} / l^{d} \mathcal{O}_{\lambda}\right)$.

For each integer $r \geq r_{0}$, put $H_{r}:=$ the image $\rho_{r}\left(\pi_{1}^{\text {geom }}\left(\mathcal{C}_{r}, c_{r}\right)\right)$ in $\operatorname{GL}\left(n, \mathcal{O}_{\lambda}\right)$. Thus $H_{r}$ is a closed subgroup of $K$. By case 1$)$, applied to the reduction mod $l^{d}$ of $\rho$, for $r \gg 0, H_{r}$ and $K$ have the same image in $\operatorname{GL}\left(n, \mathcal{O}_{\lambda} / l^{d} \mathcal{O}_{\lambda}\right)$. So by Pink's Lemma $H_{r}=K$ for all such $r$.

For b), apply Pink's Lemma to $L:=$ the image $\rho\left(\pi_{1}(W, w)\right)$ in $\operatorname{GL}\left(n, \mathcal{O}_{\lambda}\right)$ and the subgroups $J_{r}:=$ the image $\rho_{r}\left(\pi_{q}\left(\mathcal{C}_{r}, c_{r}\right)\right)$ in $\mathrm{GL}\left(n, \mathcal{O}_{\lambda}\right)$ to reduce b) to case $1)$.

For case 3$)$, use the fact $[5,9.0 .7]$ that any compact subgroup of $\operatorname{GL}\left(n, \overline{\mathbb{Q}}_{l}\right)$, in particular the image $\rho\left(\pi_{1}(W, w)\right)$, is conjugate to a closed subgroup of $\operatorname{GL}\left(n, \mathcal{O}_{\lambda}\right)$ for $\mathcal{O}_{\lambda}$ the ring of integers in some finite extension $E_{\lambda}$ of $\mathbb{Q}_{l}$ to reduce to case $2)$.

As an immediate consequence of case 3) of Lemma 6, we get the following result of Bertini type.

Corollary 7. Let $k$ be a finite field, $W / k$ a smooth, geometrically connected $k$ scheme, and $w$ a geometric point of $W$. Suppose given an integer $r_{0} \geq 1$, and, for each integer $r \geq r_{0}$, a smooth, geometrically connected $k$-scheme $\mathcal{C}_{r} / k$ and a k-morphism

$$
f_{r}: \mathcal{C}_{r} \rightarrow W
$$

which is surjective on $k_{r}$-valued points. For each $r \geq r_{0}$, pick a geometric point $c_{r}$ in $\mathcal{C}_{r}$, and a "chemin" from $f_{r}\left(c_{r}\right)$ to $w$. Let $l$ be a prime number, and $\mathcal{F}$ a lisse $\overline{\mathbb{Q}}_{l}$-sheaf on $W$ of rank denoted $n$, corresponding to a continuous homomorphism

$$
\rho: \pi_{1}(W, w) \rightarrow \operatorname{GL}\left(n, \overline{\mathbb{Q}}_{l}\right) .
$$

Denote by $G_{\text {geom, } \mathcal{F}}$ on $W$ the Zariski closure of $\rho\left(\pi_{1}^{\text {geom }}(W, w)\right)$ in $\operatorname{GL}(n) \otimes \overline{\mathbb{Q}}_{l}$. Then for $r$ sufficiently large, the pullback sheaf $\left(f_{r}\right)^{*}(\mathcal{F})$ on $\mathcal{C}_{r}$ has the same $G_{\text {geom }}$ :

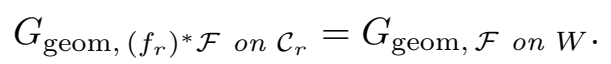

Moreover, if $\mathcal{F}$ on $W$ has the property that $\rho\left(\pi_{1}(W, w)\right)$ lies in $G_{\text {geom, } \mathcal{F} \text { on } W}\left(\overline{\mathbb{Q}}_{l}\right)$, then for $r$ sufficiently large the pullback sheaf $\left(f_{r}\right)^{*}(\mathcal{F})$ on $\mathcal{C}_{r}$ has the same property, that $\rho\left(\pi_{1}\left(\mathcal{C}_{r}, c_{r}\right)\right)$ lies in $G_{\text {geom, }}\left(f_{r}\right) * \mathcal{F}$ on $\mathcal{C}_{r}\left(\overline{\mathbb{Q}}_{l}\right)$.

Theorem 8. Let $k$ be a finite field, $X / k$ smooth and quasi-projective and geometrically connected, of dimension $n \geq 1$. Let $E / k$ be a finite extension. There exists a smooth, geometrically connected curve $C_{0} / k$, and an immersion $\pi: C_{0} \rightarrow X$ which is bijective on E-valued points. 
Proof. First apply Lemmas 2 and 3 to find an open set $V$ in $X$ which contains all the $E$-valued points and which admits an etale map $\pi$ to $\mathbb{A}^{n} \otimes k$. Let $d:=$ degree $(E / k)$, so $E$ is $k_{d}$. For each $r \geq 1$, use Lemma 1 to find a smooth, geometrically connected curve $C_{r d} / k$ in $\mathbb{A}^{n} \otimes k$ which is spacefilling for $k_{r d}$. Take $D_{r d} / k$ in $V$ to be the fibre product

$$
D_{r d}:=C_{r d} \times_{\mathbb{A}^{n} \otimes k} V .
$$

By Lemma 4, for large $r$ this closed subscheme $D_{r d}$ of $V$ is a smooth, geometrically connected curve over $k$ which is spacefilling for $k_{r d}$. Taking the $\operatorname{Gal}\left(k_{r d} / k_{d}\right)$-invariants on both sides of the equality $D_{r d}\left(k_{r d}\right)=V\left(k_{r d}\right)$, we get $D_{r d}\left(k_{d}\right)=V\left(k_{d}\right)$, or in other words $D_{r d}$ is spacefilling in $V$ for $E$. The composite inclusion $D_{r d} \subset V \subset X$ is the desired immersion.

Corollary 9. Let $k$ be a finite field, $X / k$ projective, smooth, and geometrically connected, of dimension $n \geq 1$. Let $E / k$ be a finite extension. There exists a proper, smooth, geometrically connected curve $C / k$, and a $k$-morphism $\pi: C \rightarrow X$ which is surjective on E-valued points. Moreover,

1) there is an open dense set $U$ in $C$ such that $\pi \mid U: U \rightarrow X$ is bijective on E-valued points,

2) $\pi$ is birationally an isomorphism of $C$ with its image $\pi(C)$ taken with the induced reduced structure.

Proof. Apply Theorem 8 to get $\pi: C_{0} \rightarrow X$, and then take $C / k$ to be the complete nonsingular model of $C_{0} / k$. Take $U$ to be $C_{0}$. Because $X / k$ is proper, the map $\pi$ extends to a $k$-morphism $\bar{\pi}: C \rightarrow X$ with all the asserted properties.

Question 10. Given $X / k$ projective, smooth, and geometrically connected of dimension $n \geq 2$, and $E / k$ a finite extension, is there always a closed subscheme $Y$ in $X, Y \neq X$, such that $Y(E)=X(E)$ and such that $Y / k$ is smooth and geometrically connected? What, if any, is the obstruction to the existence of such $Y$ ? For example, take for $X$ an odd dimensional projective space $\mathbb{P}^{2 n+1}$, $n \geq 1$ with homogeneous coordinates $X_{i}$ and $Y_{i}$ for $i=1, \ldots, n+1$. Write $q:=\operatorname{Card}(E)$ and take for $Y$ the smooth hypersurface $\operatorname{Hyp}(2 n+1, q)$ of degree $q+1$ :

$$
\operatorname{Hyp}(2 n+1, q): \sum_{i}\left(X_{i}\left(Y_{i}\right)^{q}-\left(X_{i}\right)^{q} Y_{i}\right)=0 .
$$

But what to do for $\mathbb{P}^{2 n}$ ? Take the "easy" case $k=E\left(=\mathbb{F}_{q}\right)$. One idea is to view $\mathbb{P}^{2 n}$ as an $\mathbb{F}_{q}$-rational hyperplane section $L=0$ of $\mathbb{P}^{2 n+1}$, and then take its $Y$ to be $L \cap \operatorname{Hyp}(2 n+1, q)$. This idea does not work, because the Gauss map for $\operatorname{Hyp}(2 n+1, q)$ is

$$
\left(X_{i}, Y_{i}\right)^{\prime} s \mapsto\left(\left(Y_{i}\right)^{q},-\left(X_{i}\right)^{q}\right)^{\prime} s=\operatorname{Frob}_{q}\left(\left(Y_{i},-X_{i}\right)^{\prime} s\right) .
$$

The map

$$
\left(X_{i}, Y_{i}\right)^{\prime} s \mapsto\left(Y_{i},-X_{i}\right)^{\prime} s
$$


is an involution of $\operatorname{Hyp}(2 n+1, q)$. Thus $\operatorname{Hyp}(2 n+1, q)$ is its own dual variety, cf., [8, XVII, 3.4]. Exactly because $\operatorname{Hyp}(2 n+1, q)$ contains all the $\mathbb{F}_{q \text {-valued points }}$ in $\mathbb{P}^{2 n+1}$, there are no $\mathbb{F}_{q}$-rational hyperplanes $L$ in $\mathbb{P}^{2 n+1}$ which are transverse to $\operatorname{Hyp}(2 n+1, q)$ !

The simplest form of the question is this: in $\mathbb{P}^{2} / \mathbb{F}_{q}$, is there a smooth plane curve $C / \mathbb{F}_{q}$ which goes through all the $\mathbb{F}_{q}$-points of $\mathbb{P}^{2}$ ?

\section{Applications to abelian varieties and to zeta functions of curves}

Theorem 11. Let $k$ be a field, $A / k$ an abelian variety of dimension $g \geq 1$. There exists a proper, smooth, geometrically connected curve $C / k$, a $k$-valued point $O_{C}$ in $C(k)$, and a $k$-morphism

$$
\pi: C \rightarrow A
$$

which maps the point $O_{C}$ on $C$ to the origin $O_{A}$ on $A$, and whose Albanese map

$$
\begin{gathered}
\operatorname{Alb}(\pi): \operatorname{Alb}\left(C / k, 0_{C}\right) \rightarrow A \\
\| \\
\operatorname{Jac}(C / k)
\end{gathered}
$$

is surjective. Moreover, if the field $k$ is infinite, there exists such data with $\pi$ a closed immersion.

Proof. We first treat the well known case when the field $k$ is infinite. The proof we give in this case (cf., $[6,10.1]$ for a variant) is quite simple. We give it both for the reader's convenience and because it conceivably could be made to work over a finite field as well, see Question 13 below. It depends on the following geometric fact:

Lemma 12. In $\mathbb{P}^{N}$ over an infinite field $k$, let $X / k$ be a closed subscheme which is smooth and geometrically connected, of dimension $n \geq 1$. Given an point $P$ in $X(k)$ and an integer $d \geq 2$, there exists a hypersurface $H / k$ of degree $d$ in $\mathbb{P}^{N}$ such that $P$ lies on $H$ and such that $X \cap H$ is smooth of dimension $n-1$.

Proof. Denote by $\mathcal{H}$ the projective space of all degree $d$ hypersurfaces in $\mathbb{P}^{N}$. Inside $\mathcal{H}$, we have two subvarieties of particular interest:

1) the "dual variety" $\check{X}$ (of $X$ for the $d$-fold Segre embedding, cf., [8, XVII, 2.4]), consisting of those degree $d$ hypersurfaces $H$ such that $X \cap H$ fails to be smooth of dimension $n-1$.

2) the hyperplane $\check{P}$ consisting of those degree $d$ hypersurfaces which contain $P$.

We claim that $\check{P}-\check{P} \cap \check{X}$ has a $k$-point. Since $\check{P}-\check{P} \cap \check{X}$ is open in the projective space $\check{P}$ and the field $k$ is infinite, $\check{P}-\check{P} \cap \check{X}$ is either empty or it has a $k$-point. [This comes down to the fact that if a $k$-polynomial in some number $m$ of variables vanishes on $k^{m}$ then it is the zero polynomial, provided $k$ is infinite.] If $\check{P}-\check{P} \cap \check{X}$ is empty, then $\check{P} \subset \check{X}$. But the dual variety is irreducible of codimension at least one, cf., [8, XVII, 3.1.4], so $\check{P}=\check{X}$. Take homogeneous 
coordinates $X_{0}, \ldots, X_{N}$ in which the point $P$ is $(1,0,0, \ldots, 0)$. The hypersurface $\left(X_{0}\right)^{d}=0$ lies in $\check{X}$ but not in $\check{P}$, contradiction.

To exhibit a $g$-dimensional abelian variety $A$ over an infinite field $k$ as the quotient of a Jacobian, embed $A$ in projective space, pick $g-1$ integers $d_{i} \geq 2$, and successively intersect $A$ with general hypersurfaces of degrees $d_{i}$ defined over $k$ which each contain the origin $0_{A}$, to obtain a smooth curve $C / k$ in $A$, defined over $k$, which contains $0_{A}$. The "weak Lefschetz theorem" [7, VII, 7.1] on hypersurface sections tells us that for any prime $l$ invertible in $k$, the restriction map

$$
H^{i}\left(A \otimes_{k} \bar{k}, \mathbb{Q}_{l}\right) \rightarrow H^{i}\left(C \otimes_{k} \bar{k}, \mathbb{Q}_{l}\right),
$$

is bijective for $i=0$, so $C / k$ is geometrically connected, and injective for $i=1$. This injectivity for $i=1$ implies that the Albanese map

$$
\operatorname{Alb}\left(C, 0_{A}\right) \rightarrow A
$$

is surjective.

The proof we give below, over a finite field, is due to Ofer Gabber. We do not know if the proof given above in the infinite field case can be made to work over a given finite field, say by taking the degrees $d_{i}$ quite large, cf., Question 13 below.

Pick a prime number $l \neq p$, and a finite extension $E / k$ such that each of the $l^{2 g}$ points in $A(\bar{k})$ of order dividing $l$ lies in $A(E)$. Apply the previous corollary to produce a proper smooth geometrically connected curve $C / k$, an open set $U \subset C$, and a $k$-morphism

$$
\pi: C \rightarrow A
$$

such that $\pi \mid U$ is bijective on $E$-valued points: $U(E) \cong A(E)$ by $\pi$. Taking $\operatorname{Gal}(E / k)$-invariants, we see that $U(k) \cong A(k)$ by $\pi$. Take $0_{C}$ in $U(k)$ to be $(\pi \mid U)^{-1}\left(0_{A}\right)$.

The image of $\operatorname{Alb}\left(C / k, 0_{C}\right)$ in $A$ is an abelian subvariety $B \subset A$. So $B(\bar{k})$ is a subgroup of $A(\bar{k})$. Hence $B(\bar{k}) \cap A(\bar{k})[l]=B(\bar{k})[l]$. But by construction we have

$$
A(\bar{k})[l] \subset A(E) \cong \pi(U(E)) \subset \pi(C(\bar{k})) \subset B(\bar{k}) .
$$

Therefore $B(\bar{k})[l]=A(\bar{k})[l]$, hence $\#(B(\bar{k})[l])=l^{2 g}$. Therefore $B$ has dimension $g$, so it must be all of $A$.

Question 13. Suppose we are in the setting of Lemma 12, but over a finite field $k$. Thus in $\mathbb{P}^{N}$ over $k$, we are given a closed subscheme $X / k$ which is smooth and geometrically connected, of dimension $n \geq 1$. Given a point $P$ in $X(k)$, does there exist an integer $d \geq 2$ and a hypersurface $H / k$ of degree $d$ in $\mathbb{P}^{N}$ such that $P$ lies on $H$ and such that $X \cap H$ is smooth of dimension $n-1$ ? Does this hold for all $d \gg 0$ ?

Corollary 14. Given a finite field $k$, and an abelian variety $A / k$, there exists a proper, smooth, geometrically connected curve $C / k$ such that the characteristic polynomial of Frobenius on $\left(H^{1}\right.$ of) $A / k$ divides the characteristic polynomial of Frobenius on ( $H^{1}$ of) $C / k$. 
Proof. Once the Albanese map is surjective, for $l \neq p$ we have a $\operatorname{Gal}(\bar{k} / k)$ equivariant inclusion

$$
H^{1}\left(A \otimes_{k} \bar{k}, \mathbb{Q}_{l}\right) \subset H^{1}\left(\operatorname{Alb}\left(C / k, 0_{C}\right) \otimes_{k} \bar{k}, \mathbb{Q}_{l}\right)=H^{1}\left(C \otimes_{k} \bar{k}, \mathbb{Q}_{l}\right),
$$

whence a divisibility of characteristic polynomials

$$
\operatorname{det}\left(1-T F_{k}\left|H^{1}\left(A \otimes_{k} \bar{k}, \mathbb{Q}_{l}\right)\right| \operatorname{det}\left(1-T F_{k} \mid H^{1}\left(C \otimes_{k} \bar{k}, \mathbb{Q}_{l}\right)\right) .\right.
$$

Corollary 15. Suppose we are given an integer $r \geq 1$, a list of $r$ Weil numbers $\alpha_{i}$ for $q:=\# k$ (each $\alpha_{i}$ is an algebraic integer which has all its archimedean absolute values equal to $\operatorname{Sqrt}(q))$, and a list $r$ positive integers $n_{i}$. There exists a proper, smooth, geometrically connected curve $C / k$ whose zeta function has a zero of multiplicity at least $n_{i}$ at the point $T=1 / \alpha_{i}$ for each $i=1, \ldots, r$.

Proof. By Honda-Tate $([3,9])$, there exists an abelian variety $A_{i} / k$ on which $\alpha_{i}$ is an eigenvalue of Frobenius. Apply the previous corollary to the product abelian variety $\prod_{i}\left(A_{i}\right)^{n_{i}}$.

\section{References}

[1] P. Deligne, Application de la formule des traces aux sommes trigonométriques, in Co-

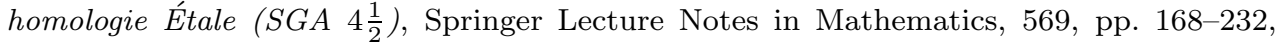
Springer-Verlag, Berlin-New York, 1977.

[2] V. Drinfeld, and S.G. Vladut, The number of points of an algebraic curve, Functional Anal. App. 17 (1983), 53-53.

[3] T. Honda, Isogeny classes of abelian varieties over finite fields, J. Math. Soc. Japan 20 (1968), 83-95.

[4] N. Katz, Exponential sums and differential equations, Annals of Mathematics Studies, 124, Princeton University Press, Princeton, NJ, 1990.

[5] N. Katz and P. Sarnak, Random matrices, Frobenius eigenvalues, and monodromy, American Mathematical Society Colloquium Publications, 45, American Mathematical Society, Providence, RI, 1999.

[6] J.S. Milne, Jacobian varieties, in Arithmetic Geometry, (G. Cornell and J.H. Silverman, eds.), pp. 167-212, Springer, New York, 1986.

[7] Séminaire de Géométrie Algébrique du Bois-Marie 1965-66, Cohomologie l-adique et Fonctions L, Springer Lecture Notes in Mathematics, 589, Springer, New York, 1977.

[8] Séminaire de Géométrie Algébrique du Bois-Marie 1967-69, Groupes de Monodromie en Géométrie Algébrique, Springer Lecture Notes in Mathematics, 340, Springer, New York, 1973.

[9] J. Tate, Classes d'Isogénie des variétés abéliennes sur un corps fini (d'après T. Honda), Exposé 352, Séminaire Bourbaki 1968/69.

Fine Hall, Department of Mathematics, Princeton University, Princeton NJ 08544 E-mail address: nmk@math.princeton.edu 